\title{
La controversia finisecular sobre el método histórico en Alemania y Francia (1883-1908)
}

\author{
Francisco Sevillano ${ }^{1}$ \\ Universidad de Alicante \\ fsevillano@ua.es
}

RESUMEN: El propósito de este trabajo es analizar la controversia sobre el método de las ciencias sociales que ocurrió en Alemania a partir de 1883, conocida como «la disputa del método» o Methodenstreit, y en Francia desde 1903, en torno al estatus científico de la historia. Estos episodios sirven como ejemplos para responder a la pregunta de cómo avanza epistemológicamente el conocimiento científico.

\section{PALABRAS CLAVE: epistemología; metodología; Methodenstreit; Char- les Seignobos; François Simiand; Émile Durkheim.}

Turn-of-the-century controversy regarding the historical method in Germany and France, 1883-1908

ABSTRACT: The purpose of this paper is to analyse controversy regarding socialsciences method in Germany after 1883, known as "the method dispute" or Methodenstreit, and in France after 1903, over the scientific status of history. These episodes serve as examples to answer the question as to how scientific knowledge advances epistemologically.

KEY WORDS: epistemology; methodology; Methodenstreit; Charles Seignobos; François Simiand; Emile Durkheim.

CÓMO CITAR ESTE ARTÍCULO/CITATION: Sevillano, Francisco, «La controversia finisecular sobre el método histórico en Alemania y Francia (1883-1908)», Hispania, 78/258 (Madrid, 2018): 193-217. https://doi.org/103989/hispania.2018.007.

${ }^{1}$ ORCID iD: http://orcid.org/0000-0001-8550-8978. 
Quizá haya que comenzar con algunas preguntas: ¿Por qué volver a un debate académico, cuyo momento álgido ocurrió en 1903, sucediéndose hasta 1908, cuando menos? La disciplina de la historia, y en su conjunto las ciencias sociales, ¿no han dejado muy atrás ese pasado de desencuentro y polémica? La epistemología, el método y la teoría, ¿no acabaron confluyendo en muchos e importantes planteamientos y procedimientos en el conjunto de las ciencias sociales hace ya tiempo? Ante estos interrogantes, estimo que hay razones para deshacer el salto que se ha dado sobre una discusión académica que fue nodal, pues constituyó un punto de origen de la moderna epistemología en la historia y las ciencias sociales. Aquella disputa sobre el método histórico y la sociología es mencionada con frecuencia, aunque apenas se retomó hace unos pocos años con motivo del centenario de la publicación del artículo de François Simiand, «Méthode historique et Science sociale», en la Revue de Synthèse historique en 1903.

Las razones que aconsejan conocer con detalle ese debate son historiográficas, metodológicas y epistemológicas: $1^{\circ}$. Los planteamientos y las discusiones que comenzaron sobre todo con la reseña que el filósofo Henri Berr hiciera de un nuevo libro de Charles Seignobos, La méthode historique appliquée aux sciences sociales, marcaron los derroteros de las ciencias sociales en formación, al menos en el mundo académico francés, durante los treinta años siguientes, y desembocaron, dentro de la disciplina de la historia, en el nacimiento y el paulatino crecimiento historiográfico de la historia social. $2^{\circ}$. Los principales términos de ese debate atañen a cuestiones esenciales del oficio del historiador, que son recurrentes, ayer como hoy: la evidencia, la prueba y la explicación, máxime tras haberse navegado por las procelosas aguas teóricas del posmodernismo. $3^{\circ}$. El examen detallado de la fase de gestación del trabajo científico, como es el caso puntual de ese debate académico, es un «acontecimiento», y como tal es tratado en este trabajo; es decir, sirve como ejemplo en la historia de las ciencias históricas y sociales que permite responder a la cuestión de cómo avanza el conocimiento científico. Tales son los objetos de análisis en este trabajo. Al respecto, se prioriza teóricamente en este ensayo un enfoque cognitivo, buscándose respuestas a la pregunta de cómo se adquiere conocimiento del pasado de las sociedades humanas a partir de la propia práctica científica en un contexto específico².

2 Al respecto, hay que destacar las propuestas de autores como GIERE, 1988. KITCHER, 1993. Estos autores, entre otros, plantean una vía intermedia entre la filosofía de la ciencia tradicional, o "programa heredado», y el "programa fuerte» de la sociología de la ciencia, entendiendo esa alternativa como un «realismo constructivo», tanto porque se construyen entidades abstractas en la investigación a modo de modelos como porque se afirma una similitud entre estas construcciones y los sistemas reales del mundo. 


\section{DER METHODENSTREIT: LA DISPUTA SOBRE EL MÉTODO}

En 1871, Carl Menger, entonces secretario de redacción del Wiener Zeitung, periódico oficial del gobierno imperial austríaco, publicó su escrito Grundsätze der Volkswirtschaftslehre o Principios de Economía Política ${ }^{3}$. Este trabajo no sólo fue la disertación escrita con la que obtuvo la capacitación para acceder a un puesto como profesor no numerario de Economía Política en la Universidad de Viena al año siguiente, sino que fue el arranque de una viva discusión sobre el método de las ciencias sociales en el ámbito académico de lengua alemana. Entre otros planteamientos, las ideas de Menger acerca de la teoría clásica del valor de los bienes inciden en los fundamentos subjetivo e individualista de la acción, es decir, en las necesidades de los individuos según las circunstancias, y no en los bienes mismos. El valor es un juicio que se hacen los agentes económicos sobre la significación de los objetos para satisfacer sus necesidades, desechándose los presupuestos de la «escuela histórica» con la pretensión de establecer una ciencia económica abstracta de validez teórica general más allá del análisis histórico concreto ${ }^{4}$. El momento álgido de esta discusión ocurrió tras la publicación del libro Untersuchungen über die Methode der Sozialwissenschaften und der politischen ökonomie insbesondere en 1883. Esta obra (referencia de lo que empezó a llamarse la «Österreichische Schule») provocó la respuesta crítica del principal referente de los jóvenes representantes de la «Historische Schule der Nationalökonomie», Gustav Schmoller ${ }^{5}$. Como muestra este episodio, los conflictos articulan el campo de producción cultural, que se estructura como un estado de las relaciones de fuerzas entre los agentes y las instituciones implicados en la lucha, que tienden a transformar o conservar sus posiciones en esta estructura ${ }^{6}$. El conflicto genera el cambio, que ocurre como un «acontecimiento». Éste supone un punto de inflexión históricamente condicionado en un continuo juego de conflictivas interacciones. La polémica en torno a Men-

3 MENGER, 1871. Sobre la figura de C. Menger, véanse, entre otros, ALTER, 1990 y BOOS, 1986. 1973.

4 Sobre éstos y otros puntos, véanse las contribuciones reunidas en HICKS y WEBER,

5 Además de la obra de MENGER, 1883, hay que citar SCHMOLLER, 7 (1883): 239258, así como la contrarréplica a los errores del historicismo del propio MENGER, 1884. Sobre la Escuela histórica alemana, y acerca de la influencia de Schmoller en las políticas de reforma social del momento, véase GRIMER-SOLEM, 2003. El pensamiento de Schmoller fue conocido tempranamente en España; véase SCHMOLLER, 1905 (obra reed. en Granada, Comares, 2007), en cuyo segundo volumen fue traducido el mencionado artículo de 1883.

6 Esta noción de «campo» fue establecida por BOURDIEU, 246 (noviembre 1996): 865906; 2002: 9-50. Esta teoría fue desarrollada en BOURDIEU, 1992. Una buena síntesis del concepto de campo aplicado a las ciencias, puede verse en BOURDIEU, 2001. 
ger ilustra puntualmente el modo como la formación disciplinar de la economía política como ciencia social fue un proceso que sucedió a partir de cambios cognitivos concretos en torno a los hechos económicos (como es el valor de los bienes) y su evaluación metodológica. Los hechos científicos, los objetos de estudio de la ciencia, son delimitados epistemológicamente por las prácticas sociales y cognitivas en el contexto histórico del trabajo de investigación. Son momentos, no exentos de polémica y disputas, en los que se generan nuevos estilos de pensamiento en torno a objetos y métodos tenidos por más adecuados que los existentes y empleados hasta entonces.

Las controversias no sólo enfrentan diferentes identidades de grupo en el campo cultural, sino que también los miembros de las distintas comunidades científicas viven inmersos en su propia realidad intelectual y profesional de acuerdo con un estilo de pensamiento, tradición y educación específicos. Cada forma de conocer el mundo selecciona diferentes cuestiones, que conecta de acuerdo con diversas reglas y propuestas ${ }^{7}$. El estilo de pensamiento determina cada concepto, dirigiendo la percepción con la correspondiente elaboración intelectiva y objetiva de lo percibido ${ }^{8}$. Es así como unas mismas concepciones, condicionadas histórica y socialmente, se extienden transversalmente por varios ámbitos de conocimiento y acaban sufriendo las mismas tensiones. En 1883, Wilhem Dilthey publicó Einletung in die Geisteswissenschaften, una introducción a las ciencias del espíritu a la que no siguió la aparición de los subsiguientes volúmenes planificados ${ }^{9}$. Su propuesta de independizar las ciencias del espíritu de las ciencias de la naturaleza, y de dotar a aquéllas de un método hermenéutico propio de interpretación, fue adoptada por el neokantismo en el marco de las «disputas sobre el método» en Alemania durante finales del siglo XIX, que en parte trataron sobre el lugar de la historia en la clasificación de las ciencias, tanto desde el punto de vista material como formal. No obstante la variedad de planteamientos con los que distintos autores fueron perfilando sus posturas en tal debate (caso del propio Dilthey, además de otros como Windelband, Rickert, Simmel y Weber), Raymond Aron afirmó que es posible definir exactamente la noción de «crítica de la razón histórica», a la que todos ellos contribuyeron. En primer lugar, porque tuvieron un objetivo común: dilucidar la naturaleza de las ciencias históricas; en segundo término, puesto que también compartieron un mismo principio filosófico: hostiles a la filosofía tradicional de la historia, procedieron a renovar las secuelas metafísicas del idealismo con una «reflexión positiva». A partir de

7 Véase, acerca de esta última afirmación, FLECK, 17 (1929): 425-430.

8 FLECK, 1935. Thomas S. Kuhn desarrolló la noción de «paradigma» a partir del mencionado concepto de «estilo de pensamiento». Véase KUHN, 1962.

9 DILTHEY, 1883. Precisamente, Schmoller confrontó esta propuesta de Dilthey con los planteamientos de Menger. Véase SCHMOLLER, 7 (1883): 253. 
estas premisas, la cuestión fundamental de la crítica de la razón histórica fue transponer el método kantiano de manera que haga inútil la filosofía de la historia y establezca una lógica original de las ciencias históricas, diferente a la de las ciencias positivas ${ }^{10}$.

La crítica buscaba renovar el paradigma de conocimiento del mundo histórico que naciera con la obra de Leopold von Ranke y que fue llamado retrospectivamente «historicismo», en alusión a que la vida y la realidad son historia $^{11}$. En este esfuerzo por reposicionar y reafirmar el conocimiento histórico en el conjunto de las ciencias, hay que destacar la aparición del primer volumen de la Deutsche Geschichte, en 1891, de Karl Gotthard Lamprecht ${ }^{12}$. El trabajo de este historiador, relacionado con la Escuela histórica alemana a través de Wilhem Roschen, suscitó una amplia y ardua polémica. Las críticas ocurrieron sobre todo a partir de 1896 con motivo de la publicación de la segunda edición de los seis primeros tomos de la Deutsche Gechichte ${ }^{13}$. Dentro de los parámetros de la nueva Kulturgeschichte, Lamprecht rechazó el método individual y descriptivo de los neorankeanos en beneficio de un método genético que permitiese formular leyes sobre la génesis del desarrollo social. Frente al predicamento de la historia política, destacó las fuerzas psicológicas como el motor de la historia ${ }^{14}$. Karl G. Lamprecht sistematizó esta concepción de la historia cultural en su escrito Die kulturhistorische Methode, editado en 1900, y en la obra Moderne Geschichtswissenschaft, que se publicó simultáneamente en alemán e inglés tras la serie de conferencias que dictó en la Universidad de Columbia en $1904^{15}$.

10 ARON, 1969 (1938): 13.

11 Para una visión general de la historiografía alemana, hay que citar IGGERS, 1968. También JAEGER y RÜSEN, 1992. El desarrollo de la tradición historicista puede verse en el detallado estudio general de BEISER, 2011. Sobre la «crisis del historicismo», de la que hablaría por primera vez Ernst Troeltsch en 1922 respecto a sus consecuencias ideológicas y teológicas, véanse RINGER, 1969. OEXLE, 2007.

12 LAMPRECHT, 1891.

13 LAMPRECHT, 1894-1896. Entre los críticos, destacó el medievalista BELOW, LXXI (1893): 465-498, así como el historiador RACHFAHL, 83 (1896): 48-96. Las discusiones que se sucedieron tuvieron su centro en la revista Historische Zeitschrift: LAMPRECHT, LXXVII (1896): 257-261. MEINECKE, LXXVII (1896): 262-266. LENZ, LXXVII (1896): 385-447. HINZE, LXXXVIII (1987), 60-66. En medio de esta polémica, véanse LAMPRECHT, 1896; 1 (1896/1897): 75-150.

14 Existe una amplia bibliografía sobre Karl G. Lamprecht, pudiéndose citar CHICKERING, 1993; 2000: 15-29, así como SCHORN-SCHÜTTE, 1984. Véanse, más ampliamente, las contribuciones reunidas en HÜBINGER, BRUCH y GRAF, 1997. También FLÖTER, JONAS y DIESENER, 2015.

15 LAMPRECHT, 1900; 1905a; 1905b. 


\section{ECOS DEL DEBATE EN FRANCIA}

Los planteamientos de Lamprecht tuvieron mejor acogida fuera de Alemania, como fue el caso de Francia ${ }^{16}$. El análisis de cuál fue la recepción de esta polémica y de cómo se desarrolló en el ámbito académico francés reafirma la validez de la formación de estilos de pensamiento, diferenciados y en disputa, como principio general del cambio científico. Más detalladamente, la descripción de los términos de debate en Francia permite ejemplificar los mecanismos que impulsan y articulan social y cognitivamente unas y otras formas de pensamiento en conflicto.

En 1889, se publicó, con el título Études sur l'état économique de la France pendant la première partie du moyen âge, la disertación Frankreis wirtschaliche Verhältnisse im 11. Jahrhundert, que Lamprecht leyó, bajo la dirección de Roscher, en el semestre de 1877-1878 en la Universidad de Leipzigi ${ }^{17}$. Las renovadoras propuestas sobre la historia fueron difundidas y debatidas en una nueva publicación periódica, Revue de Synthèse historique, que fundara el filósofo Henri Berr con un carácter interdisciplinar ${ }^{18}$. El editorial del primer número de la revista, «Sur notre programme», muestra no sólo sus propósitos, sino las ideas que serán esgrimidas contra la concepción y la práctica de la historia política ${ }^{19}$. Este editorial comenzaba con la afirmación de que la Revue de Synthèse historique respondía a una necesidad, que la publicación pretendía cubrir con ciertos contenidos. En sus primeros números, destacarían los estudios teóricos, puesto que se deseaba publicar una serie de artículos sobre el método de las diversas ciencias históricas. Ninguna ciencia podía ser abandonada a la rutina y al empirismo. Si la teoría no hace, en general, más que sancionar la práctica, la preocupación por llevar a buen puerto la teoría podría hacer progresar la práctica ${ }^{20}$.

16 Entre los primeros ecos de la discusión, hay que citar PIRENNE, LXIV (mayo-agosto 1896): 50-57, en este mismo número de la revista se publicó una reseña de la segunda edición de la Deutsche Geschichte de Lamprecht a cargo de BLONDEL, LXIV (mayo-agosto 1896): 145-159; LXV (septiembre-diciembre 1987): 323-333. Más ampliamente Célestin Bouglé expuso la situación de disciplinas como la psicología de los pueblos, la sociología, la economía política y el derecho a través de las figuras de M. Lazarus, G. Simmel, A. Wagner y R. von Ihering. Véase BOUGLÉ, 1896. Sobre C. Bouglé, véase VOGT, 20/1 (1979): 123-139.

17 La edición original en alemán de esta disertación fue publicada en LAMPRECHT, 1878, traducida al francés en 1889.

18 Véanse, particularmente, las contribuciones al número monográfico «Henri Berr et la culture du XXème siècle», entre las que hay que destacar CASTELLI, 117/1-2 (marzo 1996): 21-38. PROCHASSON, 117/1-2 (marzo 1996): 61-79. REVEL, 117/1-2 (marzo 1996): 157172. FUGLER, 117/1-2 (marzo 1996): 173-188.

19 «Sur notre programme», I/1 (agosto 1900): 1-8.

20 «Sur notre programme», I/1 (agosto 1900): 1-2. 
Pero se destacaría en la revista sobre todo lo tocante a la psicología histórica21. En este punto, la pregunta que se formuló en el editorial fue cuál era la relación de la síntesis histórica, de esa psicología a la que aspiraba la nueva revista, con la sociología. Por un lado, se afirmó que el rápido progreso de la sociología se había debido sobre todo a la excelente idea de que lo social forma parte de la historia, de que la solidaridad social es un elemento de explicación histórica. Sin embargo, sin negar el interés que pudieran tener ciertas consideraciones y especulaciones de filosofía social, la sociología debía ser un estudio positivo de lo que es social en la historia, partiendo de los datos históricos concretos, como había hecho Durkheim y su grupo mediante la aplicación de un método preciso, experimental, comparativo a los hechos históricos concretos. La sociología positiva estaría presente en parte en la publicación a través de los colaboradores de la revista Année sociologique, que fundara Émile Dukheim dos años antes, en $1898^{22}$. Por su parte, el historiador debía estudiar lo social, que se encuentra en todo y siempre, y sus posibles etapas, que se reproducen en la evolución de las sociedades. Pero era necesario igualmente que el historiador se atenga en cierta medida a las particularidades individuales que diferencian la historia y mediante las que se explican incluso las trasformaciones más generales de las sociedades. Así, la síntesis histórica no había de embrollar lo que comenzaba a desenredarse, sino llevar conjuntamente a los diversos equipos a cumplir mejor con su propia tarea y a ayudarse mutuamente a concebir con más claridad la obra común. Estas diversas tareas que unificaba la síntesis histórica debían desembocar en la psicología. El estudio comparativo de las sociedades debía llevar a la psicología social, al conocimiento de las necesidades naturales a las que responden las instituciones y de sus cambiantes necesidades. El estudio de las series históricas debían conducir a la psicología de los grandes hombres de acción y de pensamiento, de las individualidades étnicas, de los momentos críticos de la historia. Una cuestión de psicología importante y delicada era dilucidar qué papel juega el elemento individual en la historia. El entendimiento del pasado, pero también de la dirección del porvenir, dependía de la elaboración de esta psicología histórica ${ }^{23}$. Si bien el editorial concluía con la aseveración de que no hay ciencia más que de lo general, el análisis y la síntesis son inseparables. Si la síntesis debía hacer el análisis más eficaz, más consciente, el rigor de los trabajos analíticos subsistía en las tentativas de síntesis'24.

La declaración programática de la Revue de Synthèse historique puede entenderse como una «matriz» que conformó, a modo de molde, los términos,

21 «Sur notre programme», I/1 (agosto 1900): 2.

22 «Sur notre programme», I/1 (agosto 1900): 3-4.

23 «Sur notre programme», I/1 (agosto 1900): 5-6.

24 «Sur notre programme», I/1 (agosto 1900): 7. 
los derroteros y los protagonistas de la discusión subsiguiente sobre la relación entre la historia, las ciencias sociales y las ciencias de la naturaleza; el objeto de estudio en la sociedad y el pasado del hombre; la metodología científica; y el principio de causalidad y la formulación de leyes generales. Al respecto, un punto de referencia fue la disputa sobre el método que estaba sucediendo en el ámbito de las ciencias históricas en Alemania. Así, en el primer número de la revista se publicó un artículo de Karl Lamprecht, «La méthode historique en Allemagne» ${ }^{25}$. En ese mismo número inicial, una breve nota, que se publicó en el apartado «Notes, questions et discussions», dio noticia de la creación de la Kulturwissenschaftliche Gesellschaft en Friburgo Brisgovia. El apunte indicaba que sólo se habían publicado dos de las discusiones habidas en esa sociedad y que una de ellas había sido el discurso inaugural de Heinrich Rickert, Kulturwissenschaft und Naturwissenschatf ${ }^{26}$. Sin más comentarios sobre el contenido de esta conferencia, la nota precisaba que la expresión «Kulturwissenschaft» era equivalente a la de «Synthèse historique» a propósito de contemplarse las relaciones de las ciencias históricas con las ciencias de la naturaleza y abogarse por la interacción disciplinar entre la teología, la jurisprudencia, la historia, la filología, la economía social e incluso quizá hasta la filosofía en bases a sus intereses comunes ${ }^{27}$. De hecho, en el número quinto de la Revue de Synthèse historique, editado en 1905, se publicó el artículo de Rickert, «Les quatre modes de l' «Universel» en histoire» ${ }^{28}$.

En ese mismo número, otra nota publicada también en la sección miscelánea de cuestiones y discusiones daba noticia de una comunicación que el director de la revista, Henri Berr, había impartido en una reciente sesión de la Société d'Histoire moderne, relativa a la evolución de los estudios sobre la teoría de la historia en Alemania ${ }^{29}$. Berr distinguía una primera etapa en torno a 1890, en la que el episodio principal fue la lucha entre Schäfer y Gothein, el Schäfer-Gotheinsche Streit, disputándose acerca de la historia política y la Kulturgeschichte. En un segundo período, se produjo la geschichtswissenschfliche Streit a raíz de los debates provocados por la publicación del primer volumen de la Deutsche Geschichte de Lamprecht. Si bien ese primer volumen se publicó en 1891, la lucha se desató sobre todo en 1896 con motivo de

25 LAMPRECHT, I/1 (agosto 1900): 21-27. La bibliografía sobre metodología que apareciera tras la primera edición de la Deutsche Geschichte, que Lamprecht publicó en el prefacio de la tercera edición de esta obra en 1896-1900 (compuesta de 100 referencias, de las que 77 eran en lengua alemana), fue reproducida en la Revue de Synthèse historique, III-3/9 (diciembre 1901): 350-357; IV-1/10 (febrero 1902): 107-109.

26 RICKERT, 1899.

27 «Notes, questions et discussions», I/1 (agosto 1900): 111-113.

28 RICKERT, II-2/5 (abril 1901): 121-140.

29 «Notes, questions et discussions», X-1/28 (agosto 1905): 369-372 
los estudios críticos de Rachfahl y Meinecke. Esta disputa se caracterizó por la lucha entre la antigua concepción «individualista» y una nueva concepción «colectivista» de la historia. La tercera etapa comenzó a partir de 1900, coincidiendo con la fundación de la Revue de Synthèse historique en Francia. Este nuevo período no se centró tanto en las concepciones individuales de ciertas personalidades como en la teoría de la historia misma ${ }^{30}$. En opinión de Henri Berr, las preocupaciones teóricas en esos debates habían traído consigo la multiplicación de los estudios sobre la historia de las teorías, en los que la influencia de Lamprecht había sido considerable y las universidades participan cada vez más activamente ${ }^{31}$. Pero destacó sobre todo lo chocante que resultaba la participación de los filósofos y la naturaleza de su intervención. En Francia, los filósofos que se ocupaban de la historia buscaban darle un carácter científico, considerando en general la historia tradicional como un trabajo preparatorio. Sin embargo, en Alemania algunos «filósofos-sociólogos» con algunos historiadores «reformistas» aspiraban a la ciencia (como era el caso de Lamprecht, entre otros), pero para la mayoría de los filósofos alemanes la lógica de la historia arraigaba en «lo dado» de la historia tradicional. Éstos tomaban como ejemplo a los grandes historiadores del pasado, considerándose a Ranke como el maestro, y oponían la historia a la ciencia, sobre todo las ciencias de tipo «naturalista» (Naturwissenschaften) a las ciencias de un tipo especial (Geisteswissenschaften, Kulturwissenschaften, Gesichichtswissenschaften). Estos filósofos (entre los que señalaba a Dilthey, Windelband o Rickert) desarrollaban esta oposición poniendo a un lado la preocupación por lo general, la necesidad y la causalidad, y, a otro, lo individual, la contingencia, la finalidad. Según Berr, todos ellos estaban de acuerdo con los «historiadores historizantes», como Eduard Meyer, y concluía que el principal peligro de esta orientación era el riesgo de retornar a la filosofía idealista de la historia ${ }^{32}$.

Esta mirada retrospectiva a aquella disputa en Alemania, cuyos ecos resonaban en el mundo académico francés, permite subrayar la atención singular que suscitó la obra y el papel de Karl Lamprecht en la pelea por el método desde la óptica francesa, así como destacar el rechazo particular que suscitó la recepción del neokantismo alemán y, más ampliamente, la primacía de la escuela histórica alemana ${ }^{33}$. Pero las opiniones de Henri Berr al respecto

30 «Notes, questions et discussions», X-1/28 (agosto 1905): 369-370.

31 «Notes, questions et discussions», X-1/28 (agosto 1905): 370-371.

32 «Notes, questions et discussions», X-1/28 (agosto 1905): 371-372.

33 Hay que enmarcar la recepción de la disputa del método que estaba produciéndose en el ámbito académico e intelectual de Alemania en un clima más amplio de «obsesión» por lo alemán y la «cosa alemana» que no cesó de marcar la vida intelectual francesa tras el desenlace de la guerra fraco-prusiana en 1871 hasta el estallido de la Gran Guerra en el verano de 1914, como destacó DIGEON, 1959. Este referente explica como las particularidades de cualquier «estilo nacional», sea historiográfico o de otra área de conocimiento, como ocurrirá con 
no sólo muestran en qué aspectos la escuela histórica alemana y la escuela positiva francesa eran «inconmensurables» como paradigmas de conocimiento $^{34}$. En medio de los debates que estaban produciéndose también en Francia (la respuesta de Paul Lacombe al artículo de Rickert en el número séptimo de la Revue de Synthèse historique no sólo corresponde a los intereses programáticos de la revista, sino que ilustra detalladamente en base a qué términos se rechazó el neokantismo), esa visión de la polémica en Alemania permite entresacar in situ los mecanismos que impulsan el cambio científico: (1) el «ruido» externo o interferencias que provocan perturbaciones en un campo de conocimiento - como fue la reacción que ocurrió en Francia ante la penetración del Methodenstreit-; (2) la «autorreferencialidad» o relación que se establece, mediante un juego de oposiciones y afirmaciones entre grupos científicos, con una tradición intelectual ${ }^{35}$; $y$, como consecuencia de estos mecanismos, (3) el proceso de «continuidad retroactiva» en una comunidad intelectual, es decir, la alteración del conocimiento previamente establecido, añadiendo nuevas ideas o modificando o reinterpretando las ya existente. De este modo, la formación de una nueva forma de conocimiento conlleva la redefinición del ámbito científico correspondiente, aunque ello no supone siempre una ruptura ni inmediata ni absoluta con el estado del conocimiento anterior. El resultado del cambio científico no deja de ser en parte acumulativo y paulatino, sobre todo al mantenerse una determinada tradición científica esencial en la base de un ámbito de conocimiento; es normal la continuidad y el desarrollo gradual de los procedimientos instrumentales y metodológicos del trabajo investigador, más evolutivos que rupturistas, sobre todo en contraste con la teoría. El proceso de cambio puede alcanzar un punto de inflexión conforme se produce un desplazamiento desde los aspectos metodológicos hacia las cuestiones teóricas, pero sobre todo cuando un «acontecimiento» en este proceso de cambio afecta a los fundamentos ontológicos y los principios epistemológicos de la tradición de investigación de una disciplina.

el desarrollo de la sociología en Francia, revelan el peso de los esquemas dominantes que imponen en la vida intelectual y académica las instituciones en cada Estado, provocando una «reacción» desde la pertenencia a la tradición nacional de una disciplina. Véase DUMOULIN, 59-61 (1995): 176-183.

34 Como punto de partida, asumo la definición más general que T. S. Kuhn diera de los paradigmas como las «realizaciones científicas universalmente reconocidas que, durante cierto tiempo, proporcionan modelos y soluciones a una comunidad científica» (KUHN, 1971: 13). Sobre la noción de «inconmensurabilidad» entre paradigmas, véase el capítulo X, «Las revoluciones como cambios de la concepción del mundo», de la obra citada de Kuhn.

35 Acerca de la noción de «tradición de investigación», sigo la definición que diera Larry Laudan: «una tradición de investigación es un conjunto de supuestos generales acerca de las entidades y procesos de un ámbito de estudio, y acerca de los métodos apropiados que deben ser utilizados para investigar los problemas y construir las teorías de ese dominio» (LAUDAN, 1986: 116). 


\section{DISCORDIAS EN LA ALDEA GALA: DISCIPLINAS EN COMPETENCIA Y DISPU- TAS EPISTEMOLÓGICAS}

En Francia, el debate sobre el método de la historia tuvo su origen en 1901 a partir de las críticas que suscitó la publicación de la obra La Méthode historique appliquée aux sciences sociales, de Charles Seignobos ${ }^{36}$. Este libro, que fue el resultado de un curso impartido durante tres años en el Collège libre des sciences sociales, se divide en dos partes: una primera, que trata el mismo asunto que el libro Introduction aux études historiques, publicado conjuntamente con Charles-Victor Langlois ${ }^{37}$; y una segunda, enteramente nueva, que ocupaba, en palabras del autor, un terreno intermedio entre la historia y las ciencias sociales. Seignobos afirmó que el método histórico difiere radicalmente de todos los métodos de las otras ciencias, pues, en lugar de observar directamente hechos, actúa indirectamente razonando sobre documentos. Al ser todo conocimiento histórico indirecto, la historia es esencialmente una ciencia de razonamiento, siendo su método indirecto, por razonamiento ${ }^{38}$. Así, el método de estudio indirecto por los documentos, como es el método histórico, es el único que puede emplearse en dos casos: para llegar a comprender una evolución, así como un conjunto concreto. Al respecto, como cualquier otro método científico supone dos series de operaciones: estudiar el documento para determinar cuáles han sido los hechos particulares pasados de que el documento es la huella, $y$, tras haber establecido esos hechos, agruparlos en una construcción metódica para descubrir las relaciones existentes entre ellos ${ }^{39}$.

A partir de estos presupuestos básicos, Seignobos miró hacia las ciencias que estudian los hechos sociales, es decir, los que se producen en sociedad, recordando que Auguste Comte inventó la palabra «sociología» para designar la ciencia de todos los fenómenos de la sociedad. Para Charles Seignobos, esta palabra, acuñada por filósofos, correspondía al intento de agrupar varias ramas de la ciencia que habían permanecido aisladas bajo una concepción filosófica de conjunto. La denominación «ciencias sociales» se hizo usual para indicar aproximadamente el mismo conjunto de estudios, de modo que

36 SEIGNOBOS, 1901 (puede verse una reedición reciente electrónica, con prefacio de Antoine Prost, en Lyon, ENS Éditions, 2014 [http://books.openedition.org/enseditions/487]). Hay que lamentar que no se disponga de una nueva edición en español del libro de Seignobos, que fue traducido con el mismo título en 1923. Sobre este historiador francés, véase PROST, 43 (julio-septiembre 1994): 100-118.

37 LANGLOIS Y SEIGNOBOS, 1898 (puede verse una reedición reciente electrónica, con prefacio de Antoine Prost, en Lyon, ENS Éditions, 2014 [http:// http://books.openedition.org/ enseditions/2042]). El libro fue traducido al español en LANGLOIS y SEIGNOBOS, 1913. Véase, más recientemente, LANGLOIS y SEIGNOBOS, 2003.

38 SEIGNOBOS, 1923: 8.

39 SEIGNOBOS, 1923: 9. 
habían convergido tres grupos de orígenes muy alejados, formando las «ciencias sociales»: las ciencias estadísticas, incluida la demografía; las ciencias de la vida económica; y la historia de las doctrinas y de las tentativas económi$\operatorname{cas}^{40}$. Así, las ciencias sociales se limitaban a una parte restringida de los fenómenos, siendo una amalgama desemejante formada por el estudio de los hechos y las instituciones de carácter económico, por la estadística de los actos y los procesos humanos, y por la historia de las doctrinas. El único carácter común es el estudiar fenómenos referentes a los hechos materiales de los hombres, que se trata de conocer por la observación material ${ }^{41}$.

Esta definición de las ciencias sociales hacía comprensible, según Seignobos, el porqué el método histórico era indispensable para ellas. Si bien toda ciencia social debería constituirse mediante la observación directa de los fenómenos, en la práctica la observación de los fenómenos está siempre limitada a un campo muy reducido. Para llegar a un conocimiento extenso, es necesario recurrir al procedimiento indirecto, al documento, que hay que estudiar mediante el método histórico. Asimismo, toda ciencia social se aplica a fenómenos que no permanecen constantes, de modo que hay que conocer su evolución para comprenderlos. El necesario estudio de los fenómenos sociales anteriores no es posible más que con un método histórico ${ }^{42}$.

Los comentarios acerca de esta obra de Seignobos, como fue la reseña de Henri Berr en el número 12 de la Revue de Synthèse historique, publicado en 1902, generaron una viva discusión ${ }^{43}$. Berr criticó que Seignobos sólo prestara atención a los individuos, los eventos y los hechos, sobre todo políticos, rechazando las abstracciones para incidir en los motivos individuales y en la prevalencia del método psicológico de la historia. En su opinión, Seignobos contemplaba la solidaridad de los lazos humanos sin prestar atención a la noción de lo social. Al negar la historia como ciencia, no veía más que lo subjetivo en la materia y la interpretación de la historia. Estos argumentos críticos fueron desarrollados por François Simiand, estudiante de Henri Bergson y de Émile Durkheim, colaborador de la revista Année sociologique, que por entonces se encargaba de la creación de una biblioteca de ciencias sociales en el Ministerio de Comercio ${ }^{44}$. En 1903, publicó dos artículos en sendos números consecutivos de la Revue de Synthèse historique, el número 16 y el 17, en los que hizo un largo comentario de dos obras recientes, de los historiadores La-

40 SEIGNOBOS, 1923: 9ss.

41 SEIGNOBOS, 1923: 14-15.

42 SEIGNOBOS, 1923: 15-16.

43 BERR, IV-3/12 (junio 1902): 294-302.

44 Véanse las contribuciones pluridisciplinares reunidas en GILLARD y ROSIER, 1996, así como FROBERT, 2000 y STEINER, 2005. Sobre la revista L'Année sociologique, véase CLARK, 9/1 (mayo 1968): 72-91. 
combe y Seignobos respectivamente ${ }^{45}$. La vorágine de la discusión que desató, en un juego de negaciones y afirmaciones, sumando más participantes en la controversia, tuvo su foco de difusión inicial no tanto en el ámbito universitario como en una nueva revista y el procedimiento de las reseñas críticas, para acabar desplazándose hacia las intervenciones y los debates personales en seminarios organizados en espacios como la Société française de philosophie, que los difundió a través de su boletín. El desarrollo de una nueva forma de conocimiento en el campo cultural se articula siempre, como ilustra este caso, a pequeña escala como una red de cadenas de encuentros y desencuentros locales entre individuos, que se relacionan mediante «cadenas rituales de interacción ${ }^{46}$. Los rituales de interacción distintivos generan un compromiso emocional de los individuos con ciertos símbolos — cosificados como «objetos sagrados»-, y un sentimiento de constreñimiento de grupo. Una cadena de rituales de interacción se produce cuando los individuos se mueven por esa trama de encuentros y generan sus propias historias de participación ritual. Cada persona adquiere, así, su repertorio personal de símbolos con significación ritual, lo que constituye su capital cultural ${ }^{47}$. El mundo intelectual se revela como una conversación que pone en circulación el capital cultural por medio de rituales de interacción cara a cara de manera intermitente, además de mediante escritos. El intelectual participa en la charla que se da en el centro de la conversación, donde las ideas tienen la mayor sacralidad, y, si es posible, asocia su identidad a tales ideas de manera competitiva para que sus propias propuestas circulen ampliamente en la conversación y le den reputación personal ${ }^{48}$.

El conflicto es la fuente de energía de la vida intelectual al formarse, en ese juego de encuentros y desencuentros personales, las identidades de grupos enfrentados, como sucedió frente a la prevalencia que la historia había conquistado en los últimas décadas del siglo XIX en Francia ${ }^{49}$. En medio del pro-

45 SIMIAND, VI-1/16 (febrero 1903): 1-22; VI-2/17 (abril 1903): 129-157. Simiand había presentado previamente el contenido de esos otros dos textos en la Société d'Histoire moderne; véanse los resúmenes en SIMIAND, 2 (1903a): 73-77; 2 (1903b): 79. Con anterioridad, publicó ya una recensión bastante descriptiva del libro de Ch.-V. Langlois y Ch. Seignobos, Introduction aux études historiques, en SIMIAND, VI (1898): 632-641, en la que concluía con una crítica moderada de esta obra, puntualizando el problema que cabía observar, en su opinión, en relación con el vocabulario empleado, al no hablarse sin más de fenómenos sociales o no utilizarse la palabra «ley» más que calificada como «ley empírica», y afirmando que las ciencias sociales no diferían más que en grado respecto a las ciencias naturales (640641).

46 Véase COLLINS, 1998; 2004.

47 COLLINS, 1998: 29.

48 COLLINS, 1998: 30-31.

49 Sobre la autonomía y la consolidación profesional de la historia en el marco de la reforma de la enseñanza superior en la III República francesa, véase KEYLOR, 1975. 
ceso de paulatina institucionalización en el ámbito universitario, algunos miembros de la nueva especialidad científica de la sociología, en muchos casos de formación y profesión filosófica, fueron organizando su trabajo en torno a ciertos objetivos y presupuestos nuevos como un grupo coherente y activista $^{50}$. La ocupación de un lugar en el espacio intelectual, así como la consiguiente conquista del terreno profesional e institucional, se juega a través del reconocimiento y la implantación de una nueva disciplina de conocimiento $^{51}$. La delimitación de un cuerpo de conocimiento disciplinar, como ocurrió con la sociología en Francia, se desarrolla como un proceso de reorganización teórica y metateórica, tras el que subyace una ruptura epistemológica y ontológica en un ámbito de conocimiento. En segundo término, la redefinición de una tradición intelectual se convierte así en un mecanismo social importante porque levanta una barrera entre quienes aceptan los nuevos presupuestos y prácticas, y aquéllos otros que no los comparten, motivando personalmente a los miembros del grupo en formación ${ }^{52}$. En tercer lugar, este proceso va generando un fenómeno de «hibridación» disciplinar mediante la recombinación de distintos saberes, lo que conlleva una actitud innovadora al adecuarse los métodos y sus presupuestos del anterior rol del investigador en una disciplina al objeto de estudio de la nueva con el propósito de crear un nuevo rol ${ }^{53}$. Esta asociación, de complementariedad con la historia (como declaración de principios, al menos), fue central en el programa de L'Année sociologique, tal y como se declaraba en el prefacio del primer número de la revista ${ }^{54}$. Así, se afirmó que la nueva publicación apelaba sobre todo a la historia, que sólo podría ser una ciencia en la medida en que fuera explicativa, es decir, en tanto que procediese comparando ${ }^{55}$. Ahora bien, si la historia procediese mediante comparación, devendría en lo mismo que la sociología. Por su parte, ésta necesitaría la colaboración de los historiadores para poder proveerse de los hechos que le intere-

CARBONNELL, 1976. NOIRIEL, 1 (septiembre 1990): 58-85, así como el panorama general expuesto en BOER, 1998 (ed. en neerlandés de 1987).

50 Véanse, entre otras referencias, KARADY, 17-2 (abril-junio 1976): 267-311. KARADY, 20-1 (enero-marzo 1979): 49-82. LEROUX, 1998. MUCCHIELLI, 116/1 (enero 1995): 50-88; 2011.

51 SCHLANGER, 54 (1992): 291-292. Véanse, más detalladamente, las contribuciones reunidas en BOUTIER, PASSERON y REVEL, 2006.

52 Sobre el desarrollo de una especialidad científica, véase MULLINS, 10/1 (1973): 5182. GRIFFITH y MULLINS, 177 (15 septiembre 1977): 959-964.

53 BEN-DAVID y COLLINS, 31/4 (agosto 1966): 459. Esta «hibridación» estuvo favorecida por algunos cambios en el ámbito universitario de París a finales del siglo XIX: una nueva forma de clasificación intelectual, en el que los cuerpos de pertenencia eran menos importantes que las relaciones en base a la función investigadora. Véase, al respecto, CHARLES, 47-48 (junio 1983):77-89.

54 «Préface», I (1898): I-VII.

55 «Préface», I (1898): II. 
san. Lejos del antagonismo, ambas disciplinas tienden naturalmente la una hacia la otra, afirmándose que todo hacía prever que estaban llamadas a confundirse en una disciplina común, en la que sus elementos respectivos se combinarían y unificarían. Como resultado, las fórmulas explicativas de la ciencia podrían extenderse progresivamente a toda la complejidad de los hechos sociales en vez de reproducir sólo sus contornos más generales, al tiempo que la erudición histórica cobraría un sentido al emplearse en resolver los problemas más graves que se plantean a la humanidad ${ }^{56}$.

Sin embargo, el propósito de la mutua configuración interdisciplinar en una nueva ciencia social devino más bien en la práctica de estos debates en la elevación hegemónica de la naturaleza de la sociología o de la historia a un plano transdisciplinar. Tal fue el caso de Charles Seignobos en su obra, ya mencionada, La Méthode historique appliquée aux sciences sociales ${ }^{57}$. Los principales planteamientos de esa obra fueron reiterados por Seignobos en la conferencia sobre las relaciones de la sociología con la historia que dictara en la École des Hautes Études Sociales, de París, en 1904, que fueron respondidos puntualmente por Célestin Bouglé en esa misma sesión ${ }^{58}$. De este modo, la escuela durkheimiana en formación arremetió simétricamente en aquella pelea hegemónica, como atestiguan las principales afirmaciones que el propio Émile Durkheim hizo en el artículo «Sociologie et Sciences Sociales», que se había publicado en 1903. En resumen, señaló que la sociología conllevaba un cambio radical en el método y la organización de las ciencias sociales. Ello se debía a que atendía a la realidad específica de los hechos sociales, cuyas leyes son irreductibles a las de la biología o la física. La sociología había nacido en el seno de la filosofía positiva, constituyéndose lejos de las disciplinas especiales al tender a lo general y a las miradas de conjunto. Su misión era integrar las ciencias sociales particulares en su seno al hacer entrar en ellas la idea de ley

56 «Préface», I (1898): III.

57 Mi interpretación difiere del análisis que hiciera L. Mucchielli, quien afirmó que ya el libro Introduction aux études historiques, publicado por Charles-Victor Langlois y Charles Seignobos, fue una reacción frente a los sociólogos, una defensa y una crispación de la identidad amenazada de la disciplina histórica, de modo que constituyó un discurso de defensa frente a las ciencias sociales, que podría considerarse prácticamente ya en su época como un anacronismo (MUCCHIELLI, 59-61 (1995): 130-136). Creo que se trató de una reacción mutua, si bien es cierto que hubo historiadores (Paul Lacombe, por ejemplo) que iniciaron entonces un proceso de «hibridación» con ciertas ciencias sociales particulares, como la economía y la misma sociología, que condujo finalmente al nacimiento de la «nouvelle histoire». Sin embargo, no comparto la idea de Antonie Prost acerca de que hubiera dos «niveles de discurso» en este debate, encontrándose la historia fuera de la misma «longitud de onda» que el discurso de Simiand o Durkheim, pues estimo que ambos discursos histórico y sociológico fueron parejos en sus propósitos hegemónicos. Véase NOIRIEL, 2003: 47-61.

58 SEIGNOBOS y BOUGLÉ, XII (1904): 161-167. 
natural, pues un fenómeno social no emana de los sentimientos individuales de los hombres, sino que son producto de la conciencia colectiva ${ }^{59}$.

El pensamiento de Émile Durkheim subyace en estas disputas. Como un grupo altamente coherente, los sociólogos franceses se fueron organizando bajo el liderazgo de su figura, que sirvió como ejemplo científico. Durkheim puso las bases para el trabajo en la sociología francesa con su tesis doctoral, que se publicó con el título De la division du travail social: étude sur l'organisation des sociétés supérieures (Paris, Félix Alcan, 1893), pero sobre todo con la obra Les règles de la méthode sociologique (Paris, Félix Alcan, $1895)^{60}$. Precisamente, el prefacio a la segunda edición de esta obra fue publicado en el cuarto número de la Revue de Synthèse historique antes de la aparición del libro en 190161; y, exactamente, Durkheim cerró los debates con Seignobos en la sesión de la Société française de philosophie de 28 de mayo de $1908^{62}$. De este modo, Durkheim se sumó a las discusiones que inició Simiand nuevamente unos tres años antes, en la sesión de 3 de mayo de 1906, también organizado en la Société française de philosophie, en la que abordó la problemática de la causalidad y la explicación científica. Los términos de esa conferencia de François Simiand fueron respondidos por Charles Seignobos en una nueva sesión de 30 de mayo de 190763. En su intervención, incidió en las condiciones empíricas del trabajo del historiador a partir del documento y en los motivos personales conscientes como fenómenos psicológicos explicativos inmediatos de los actos humanos. Justamente, ello fue criticado por Durkhein en la tercera sesión de discusiones, que trató sobre el lugar del «inconsciente» como factor explicativo (con una intervención previa de Seignobos), puntualizándose las limitaciones que existen en las razones dadas por los propios agentes históricos como factores explicativos. Los términos de esos debates muestran la continuidad sustancial de un thema transversal en historia y en las ciencias sociales, como es la relación entre individuo y sociedad ${ }^{64}$. Pero asimismo revela el desplazamiento respecto a una común tradición de investigación a historiadores y sociólogos en Francia: la ciencia positiva. Según Auguste Comte, la positividad racional se circunscribe al dominio de la verdadera

\footnotetext{
59 DURKHEIM y FAUCONNET, 55 (mayo 1903): 465-497.

60 Hay que citar las correspondientes publicaciones de estas obras en español: DURKHEIM, 1912; 1928.

61 DURKHEIM, II/4 (1901): 3-17.

62 SEIGNOBOS, VIII (1908): 217-247, exposición de Charles Seignobos, seguida de una discusión con varios intervinientes en la sesión, como fue Émile Durkheim.

63 SIMIAND, VI (1906): 245-290. SEIGNOBOS, VII (1907): 261-299, exposición de Charles Seignobos seguida de una discusión con varios intervinientes en la sesión, entre otros François Simiand.

64 Véase HOLTON, 188/4186 (25 abril 1975): 328-334.
} 
observación, única base del conocimiento ${ }^{65}$. La regla fundamental es que toda proposición ha de reducirse estrictamente al mero enunciado de un hecho, particular o general, resultando su eficacia científica de su conformidad, directa o indirecta, con los fenómenos observados. Así, la pura imaginación pierde la supremacía y se subordina a la observación. La revolución fundamental del estado positivo o real, según Comte, era sustituir la inaccesible determinación de las causas propiamente dichas por la mera investigación de las leyes, es decir, de las relaciones constantes que existen entre los fenómenos observados ${ }^{6}$. Una segunda característica es que el estudio de los fenómenos debe permanecer siempre relativo en lugar de llegar a ser absoluto ${ }^{67}$. A ello se añade, para Comte, que la verdadera ciencia, lejos de estar formada por meras observaciones, tiende a la previsión racional ${ }^{68}$. Ello resulta de que la ciencia es conforme al principio de la invariabilidad de las leyes naturales ${ }^{69}$. El término «positivo» designa, así, lo «real», lo «útil», la «certeza» y lo «preciso» ${ }^{70}$.

¿Cuáles fueron los desplazamientos de las disciplinas de la historia y la sociología en Francia respecto a esa común tradición de investigación? La respuesta pasa por contestar a una problemática central: el concepto de evidencia y sus implicaciones ontológicas y epistemológicas, es decir, qué cuenta como una observación correcta y qué se puede inferir adecuadamente de ella ${ }^{71}$. La renovada historiografía republicana francesa resolvió esta problemática situando la herencia de la crítica documental, sobre todo recibida de la «escuela histórica» alemana, en la base del método de la ciencia positiva. Sin embargo, el equilibrio entre las prescripciones y las limitaciones documentales del conocimiento histórico y la necesidad de explicar los acontecimientos pasados de las sociedades humanadas puede constatarse, por ejemplo, en las tempranas críticas de Seignobos a la enseñanza de la historia en las universidades alemanas, pues consideraba que era excesivamente empírica y erudita ${ }^{72}$. La tradición de investigación de la ciencia positiva fue un legado común a partir del cual se fue produciendo en la historia y en las nacientes disciplinas sociales en Francia, antes y después respectivamente, un «desplazamiento» ontológico acerca del objeto de estudio y epistemológico sobre los fundamentos del conocimiento

65 COMTE, 1844: 12.

66 COMTE, 1844: 12-13.

67 COMTE, 1844: 13.

68 COMTE, 1844: 16.

69 COMTE, 1844: 18.

70 COMTE, 1844: 40-41.

71 Véase, sobre la historicidad de la problemática de la evidencia científica y la importancia de su estudio por la llamada epistemología histórica, GUILLAUMIN, 7 (2004): 51-78; 2005, así como CHANG, 2004.

72 SEIGNOBOS, 1881. En este sentido, disiento de la afirmación que hiciera Charles O. Carbonell, que han seguido otros historiadores, de que no hubo una escuela positivista en la III República francesa (CARBONELL, 21-22 (1978): 173-185). 
verdadero $^{73}$. Precisamente, en el caso de la sociología, y en particular de la escuela de Durkheim, el desplazamiento hacia lo social supuso contemplar la especificidad ontológica de los fenómenos sociales, que no tienen su sustrato en lo individual. Asimismo, conllevó negar un status privilegiado al sujeto en el acto de conocimiento, ya que las relaciones sociales son irreductibles a las relaciones intersubjetivas al no ser la voluntad de los sujetos el origen o la esencia de la sociedad. La sociedad es un objeto diferente a la naturaleza del individuo, y las relaciones sociales no son idénticas a las relaciones interpersonales. Se produjo, así, una «reificación» de lo social ${ }^{74}$. Este nuevo objeto epistémico, el «hecho social», es delimitado por las condiciones técnicas de investigación (como fue el caso de la aplicación de la estadística a los estudios sociales ${ }^{75}$ ) y los procedimientos metodológicos de evaluación, sobre todo en torno a su capacidad explicativa como principal criterio de elección de teorías ${ }^{76}$.

\section{CONCLUSIÓN}

Los ejemplos expuestos de las disputas metodológicas en Alemania desde 1883 y en Francia desde 1903, sobre todo en relación con el estatus científico de la historia, han permitido resaltar la idea de que la dinámica científica ocurre mediante un cambio cognitivo de la realidad estudiada, que constituye nuevos estilos de pensamiento, diferenciados y en disputa con los existentes. Se trata de un modelo que denominaría «morfogénesis cognitiva», fundamentado en la premisa de que subyace, en toda construcción de conocimiento, la intuición de una realidad primera de la que deriva la legitimidad del conocimiento adquirido.

En la dinámica científica, el cambio ocurre a partir de un «acontecimiento» en el proceso de adecuación entre tal intuición y su racionalización. La morfogénesis o retroalimentación es la capacidad que todo sistema de conocimiento establecido tiene de modificarse como estrategia para mantenerse viable a través de la propia práctica científica: las condiciones técnicas de investigación (como son las diferentes técnicas de investigación histórica) y

73 La propuesta a favor de un giro hacia el fundamento ontológico de la observación científica en cada contexto histórico puede verse en DASTON, 99 (2008): 97-110. DASTON y LUNBEK, 2011.

74 Véase, al respecto, HIRST, 1975. Sobre cómo se producen los procesos de reificación, véase THOMASON, 1982.

75 Las herramientas estadísticas disponibles por entonces eran sobre todo tablas de frecuencias, porcentajes y promedios. Véase DURKHEIM, 26 (1888): 446-463; 1897 (trad. Española de 1928).

76 Sobre la expresión «sistema experimental», entendida como la mínima unidad funcional de la investigación, puede verse RHEINBERGER, 34/1 (enero-junio 2011): 307321; 1997. 
los procedimientos metodológicos de evaluación, que delimitan un «objeto epistémico» de estudio. Exactamente, se han subrayado tres mecanismos que impulsan el cambio científico: (1) el «ruido» externo o interferencias; (2) la «autorreferencialidad» o relación que se establece, mediante un juego de oposiciones y afirmaciones entre grupos científicos, con una tradición intelectual; y, como consecuencia de estos mecanismos, (3) el proceso de «continuidad retroactiva» en una comunidad intelectual, es decir, la alteración del conocimiento previamente establecido, añadiendo nuevas ideas o modificando o reinterpretando las ya existentes.

\section{Bibliografía}

Alter, Max, Carl Menger and the Origins of Austrian Economics, Oxford, Westwiew Press, 1990.

Aron, Raymond, La philosophie critique de l'histoire. Essai sur une théorie allemande de l'histoire, Paris, Librairie philosophique J. Vrin, 1969 (ed. or. de 1938).

Beiser, Frederick C., The German Historicist Tradition, Oxford, Oxford University Press, 2011.

Below, Georg von, «Rezension von Karl Lamprecht, Deutsche Geschichte Band 13», Historische Zeitschrift, LXXI (Berlin, 1893): 465-498.

Ben-David, Joseph y Collins, Randall, «Social Factors in the Origins of a New Science: the Case of Psychology», American Sociological Review, 31/4 (Chicago, agosto 1966): 451-465.

Berr, Henri, «Les rapports de l'histoire et des sciences sociales d'après M. Seignobos, Revue de Synthèse historique, IV-3/12 (Paris, junio 1902): 294-302.

Blondel, Georges, Revue historique, 22éme année, LXIV (Paris, mayo-agosto 1986): 145-159.

Blondel, Georges, «Le Congrès des historiens allemands à Innsbruck et la science de l'histoire en Allemagne», Revue historique, 22éme année, LXV (Paris, septiembre-diciembre 1987): 323-333.

Boer, Pim den, History as a Profesion. The Study of History in France, 1818-1914, Princeton, NJ, Princeton University Press, 1998 (ed. or. en neerlandés de 1987).

Boos, Margarete, Die Wissenschaftstheorie Carl Mengers. Biographische und ideengenschichtliche Zusammenhänge, Wien, Böhlau, 1986.

Bouglé, Célestin, Les sciences sociales en Allemagne. Les méthodes actuelles, Paris, Félix Alcan, 1896.

Bourdieu, Pierre, «Champ intellectuel et projet créateur», Les Temps modernes, 246 (París, November 1966): 865-906 (trad. al esp. en AA.VV., Problemas estructurales, México, Siglo XXI, 1967: 135-181).

Bourdieu, Pierre, Les règles de l'art. Genèse et structure du champ littéraire, Paris, Seuil, 1992 (trad. al esp.: Las reglas del arte. Génesis y estructura del campo literario, Barcelona, Anagrama, 1995). 
Bourdieu, Pierre, Science de la science et réflexivité, Paris, Éditions Raisons d'agir, 2001 (trad. al esp.: El oficio científico. Ciencia de la ciencia y reflexividad. Curso del Collège de France 2000-2001, Barcelona, Anagrama, 2003).

Bourdieu, Pierre, Campo de poder, campo intelectual. Itinerario de un concepto, Buenos Aires, Montressor, 2002.

Boutier, Jean, Passeron, Jean-Claude y Revel, Jacques (dirs.), Qu'est-ce qu'une discipline?, Paris, Éditions de l’École des Hautes Études en Sciences Sociales, 2006.

Carbonnell, Charles O., Histoire et historiens, une mutation idéologique des historiens français, 1865-1885, Toulouse, Privat, 1976.

Carbonell, Charles O., «L'Histoire dite positiviste en France», Romantisme, 21-22 (Paris, 1978): 173-185.

Castelli Gattinara, Enrico, «L'idée de la synthèse: Henri Berr et les crisis du savoir dans la première moitié du $\mathrm{XX}^{\mathrm{e}}$ siècle», )», Revue de Synthése, 117/1-2 (Paris, marzo 1996): 21-38.

Chang, Hasok, Inventing Temperature. Measurement and Scientific Progress, New York, Oxford University Press, 2004.

Charle, Christophe, «Le champ universitaire parisien à la fin du 19ème siècle», Actes de la recherche en sciences sociales, 47-48 (Paris, junio 1983): 77-89.

Chickering, Roger, «The Lamprecht controversy», en Hartmut Lehmam (ed.), Historikerontroversen, Göttingen, Wallstein, 2000: 15-29.

Chickering, Roger, Karl Lamprecht. A German Academic Life (1856-1915), Atlantic Highland, NJ, Humanities Press, 1993.

Clark, Terry N., «The Structure and Functions of a Research Institute: The Année sociologique», European Journal of Sociologique, 9/1 (Paris, mayo 1968): 72-91.

Collins, Randall, The sociology of philosophies: a global theory of intelectual change, Cambridge \& London, The Harvard University Press, 1998 (trad. al esp.: Sociología de las filosofías. Una teoría global del cambio intelectual, Barcelona, Editorial Hacer, 2005).

Collins, Randall, Interaction Ritual Chains, Princeton \& Oxford, Princeton University Press, 2004 (trad. al esp.: Cadenas de rituales de interacción, Barcelona, Anthropos, 2009).

Comte, Auguste, Discours sur l'esprit positivif, Paris, Carilian-Goeury et $\mathrm{V}^{\text {or }}$ Dalmont, Éditeurs, 1844 (trad. al esp.: Discurso sobre el espíritu positivo, Madrid, Revista de Occidente, 1934).

Daston, Lorraine, «On Scientific Observation», Isis, 99 (Chicago, 2008): 97-110.

Daston, Lorraine y Lunbek, Elizabeth (ed.), Histories of Scientific Observation, Chicago, University of Chicago Press, 2011.

Digeon, Claude, La crise allemande de la pensé française (1870-1914), Paris, Presses universitaires de France, 1959.

Dilthey, Wilhelm, Einleitung in die Geisteswissenschaten, Leipzig, Duncker \& Humblet, 1883 (trad. al esp.: Introducción a las ciencias del espíritu, México, FCE, 1944).

Dumoulin, Olivier, «Le style national de l'historiographie», Espaces Temps, les Cahiers, 59-61 (Lausanne, 1995): 176-183. 
Durkheim, Émile, «Suicide et natalité. Étude de statistique morale», Revue philosophique, 26 (1888): 446-463 (trad. al esp.: «Suicidio y natalidad: estudio de estadística moral», Revista Española de Investigaciones Sociológicas, 81 (Madrid, 1998): 171-191).

Durkheim, Émile, Le Suicide, Paris, Félix Alcan, 1897 (trad. al esp.: El suicidio: estudio de sociología, Barcelona, Editorial Reus, 1928, reed. en Madrid, Akal, 1976).

Durkheim, Émile, «De la méthode objective en Sociologie», Revue de Synthèse historique, II/4 (Paris, 1901): 3-17.

Durkheim, Émile y Fauconnet, Paul, «Sociologie et Sciences Sociales», Revue philosophique, 55 (Paris, mayo 1903): 465-497.

Durkheim, Émile, Las reglas del método sociológico, Madrid, Daniel Jorro, 1912.

Durkheim, Émile, La división del trabajo social, Madrid, Daniel Jorro, 1928.

Fleck, Ludwik, «Zur Krise der "Wirklichkeit"», Die Naturwissenschaften, 17 (Berlin, 1929): 425-430 (trad. al esp.: «Sobre la crisis de la "realidad”», Revista Española de Investigaciones Sociológicas, 67 (Madrid, 1994): 251-261).

Fleck, Ludwik, Entstehung und Entwicklung einer wissenschaftlichen Tatsache. Einführung in die Lehre vom Denkstil und Denkkollektiv, Basel, Benno Schwabe \& Co., 1935 (trad. al esp.: La génesis y el desarrollo de un hecho científico. Introducción a la teoría del estilo de pensamiento y del colectivo de pensamiento, Madrid, Alianza Editorial, 1986).

Flöter, Jonas y Diesener, Gerald (eds.), Karl Lamprecht (1856-1915): Durchbruch in der Geschichtswissenschaft, Leipzig, Leipziger Universitätsverlag, 2015.

Frobert, Ludovic, Le travail de François Simiand (1873-1935), Paris, Economica, 2000.

Fugler, Martin, «Fondateurs et collaborateurs, les débuts de la Revue de Synthèse historique», Revue de Synthése, 117/1-2 (Paris, marzo 1996): 173-188.

Giere, Ronald N., Explaining Science. A Cognitive Approach, Chicago, The University of Chicago Press, 1988.

Gillard, Lucien y Rosier, Michel (dirs.), François Simiand (1873-1935). Sociologie. Histoire. Économie, Amsterdam, éditions des Archives Contemporaines, 1996.

Griffith, Belver C. y Mullins, Nicholas C., "Coherent Social Groups in Scientific Change. "Invisible colleges” may be consistent throughout science», Science, 177 (Washington, 15 septiembre 1977): 959-964.

Grimer-Solem, Erik, The Rise of Historical Economics and Social Reform in Germany, 1864-1894, Oxford, Clarendon Press, 2003.

Guillaumin, Godfrey, «Normativismo histórico (o la formación histórica de la normatividad científica) y el problema de la subdeterminación», Argumentos de Razón Técnica, 7 (Sevilla, 2004): 51-78.

Guillaumin, Godfrey, El surgimiento de la noción de evidencia. Un estudio de epistemología histórica sobre la evidencia científica, México, Universidad Nacional Autónoma de México, 2005.

Hicks, John Richard y Weber, Wilhelm (eds.), Carl Menger and the Austrian School of Economics, Oxford, Clarendon Press, 1973.

Hinze, Otto, «Über individualistische und kollektivistische Geschichtsauffassung», Historische Zeitschrift, LXXXVIII (Berlin, 1897): 60-66. 
Hirst, Paul Q., Durkheim, Bernard and Epistemology, London/Boston, Routledge \& Kegan Paul, 1975.

Holton, Gerald, «On the Role of Themata in Scientific Thought», Science, 188/4186 (Washington, 25 abril 1975): 328-334 (trad. al esp.: «Los “themata” en el pensamiento científico», en Ensayos sobre el pensamiento científico en la época de Einstein, Madrid, Alianza Editorial, 1982: 19-42).

Hübinger, Gangolf, Bruch, Rüdiger vom y Graf, Friedrich Wilhelm (eds.), Kulturund Kulturwissenschaften um 1900, Leipzig, Franz Steiner, 1997.

Iggers, Georg G., The German Conception of History. The National Tradition of Historical Thought from Herder to the Ppresent, Middletown, CT, Wesleyan University Press, 1968.

Jaeger, Friedrich y Rüsen, Jörn, Geschichte des Historismus: eine Einführung, München, Beck, 1992.

Karady, Victor, «Durkheim, les sciences sociales et l'Université: bilan d'un semiéchec», Revue française de sociologie, 17-2 (Paris, abril-junio 1976): 267-311.

Karady, Victor, «Stratégies de réussite et modes de faire-valoir de la sociologie chez les durkheimiens», Revue française de sociologie, 20-1 (Paris, enero-marzo 1979): 49-82.

Keylor, William R., Academy and community. The Formation of the French Historical Profession, Cambridge, Mass., Harvard University Press, 1975.

Kitcher, Philip, The Advancement of Science. Science without Legend, Objectivity witouht Illusions, Nueva York, Oxford University Press, 1993 (trad. al esp.: El avance de la ciencia, México, UNAM, 2003).

Kuhn, Thomas S., The Structure of Scientific Revolutions, Chicago, University of Chicago Press, 1962 (trad. al esp.: La estructura de las revoluciones científicas, México, FCE, 1971).

Lamprecht, Karl, Beiträge zur Geschichte des französischen Wirtschaftslebens im 11. Jahrhundert, Leipzig, Duncker \& Humblot, 1878 (trad. al francés en Paris, Alphose Picard et Guilaumin et C $C^{\text {ie }}$, 1889).

Lamprecht, Karl, Deutsche Geschichte, vol. 1, Berlin, R. Gaertner, 1891.

Lamprecht, Karl, «Zum Unterschiede der älteren und jüngeren Richtungen der Geschichtswissenschaft», Historische Zeitschrift, LXXVII (Berlin, 1896): 257-261.

Lamprecht, Karl, Alte und neue Richtungen in der Geschichtswissenschaft, Berlin, Heyfelder, 1896.

Lamprecht, Karl, «Was ist Kulturgeschichte? Beitrag zu einer empirischen Historik», Deutsche Zeitschrift für Geschichtswissenschaft, 1 (Freiburg, 1896/1897): 75-150.

Lamprecht, Karl, Deutsche Geschichte, 5 vols. (en 6 tomos), Berlin, R. Gaertner, 1894-1896.

Lamprecht, Karl, Die kulturhistorische Methode, Berlin, R. Gaertner, 1900.

Lamprecht, Karl, «La méthode historique en Allemagne», Revue de Synthèse historique, I/1 (Paris, agosto 1900): 21-27.

Lamprecht, Karl, Moderne Geschichstwissenschaft. Fünf Vortrage, Freiburg am Breisgau, Hermann Heyfelder, 1905a. 
Lamprecht, Karl, What is History? Five Lectures on the Modern Science of History, New York, The Macmillan Company, 1905b.

Langlois, Charles-Victor y Seignobos, Charles, Introduction aux études historiques, Paris, Hachette et $C^{\text {ie }}, 1898$ (puede verse una reedición reciente electrónica, con prefacio de Antoine Prost, en Lyon, ENS Éditions, 2014 [http://books.openedition.org /enseditions/2042]), trad. al esp. en Madrid, Daniel Jorro editor, 1913.

Langlois, Charles-Victor y Seignobos, Charles, Introducción a los estudios históricos, edición de Francisco Sevillano Calero, Alicante, Publicaciones de la Universidad de Alicante, 2003.

Laudan, Larry, El progreso y sus problemas. Hacia una teoría del crecimiento científico, Madrid, Ediciones Encuentro, 1986 (ed. en inglés de 1977).

Lenz, Max, «Lamprechts Deutsche Geschichte 5. Bd.», Historische Zeitschrift, LXXVII (Berlin, 1896): 385-447.

Leroux, Robert, Histoire et sociologie en France. De l'histoire à la sociologie durkheimienne, Paris, PUF, 1998.

Meinecke, Friedrich, «Erwiderung», Historische Zeitschrift, LXXVII (Berlin, 1896): 262-266.

Menger, Carl, Grundsätze der Volkwirthschaftslehre, Wien, Wilhelm Braumüller, 1871 (trad. al esp.: Principios de Economía Política, Madrid, Unión Editorial, 1983).

Menger, Carl, Untersuchungen über die Methode der Sozialwissenschaften und der politischen ökonomie insbesondere, Leipzig, Duncker \& Humblot, 1883 (trad. al esp.: El método de las ciencias sociales, Madrid, Unión Editorial, 2006).

Menger, Carl, Die Irrtümer des Historismus in der deutschen Nationalökonomie, Wien, Alfred Hölder, 1884 (trad. al esp.: El método de las ciencias sociales, Madrid, Unión Editorial, 2006).

Menger, Carl, «Grundzüge einer Klassifikation der Wirtschaftwissenschaften», Jahrbücher für Nationalökonomie und Statistik, 19-53/5 (Stuttgart, 1889): 465-496 (trad. al esp.: El método de las ciencias sociales, Madrid, Unión Editorial, 2006).

Mucchielli, Laurent, «Aux origines de la Nouvelle Histoire en France: l’évolution intellectuelle et la formation du champ des sciences sociales (1880-1930)», Revue de Synthèse, 116/1 (Paris, enero 1995): 50-88.

Mucchielli, Laurent, «Une lectura de Langlois et Seignobos», Espaces Temps, les Cahiers, 59-61 (Lausanne, 1995): 130-136.

Mucchielli, Laurent, La découverte du social. Naissance de la sociologie en France, Paris, La Découverte, 2011.

Mullins, Nicholas C., «The Development of a Scientific Specialty: The Phage Group and the Origins of Molecular Biology», Minerva, 10/1 (Dordrecht, 1973): 51-82.

Noiriel, Gérard, «Naissance du métier d'historien», Genèsses, 1 (Paris, septiembre 1990): 58-85.

Noiriel, Gérard, «L' «éthique de la discussion» chez François Simiand», en Penser avec, penser contre. Itinéraire d'un historien, Paris, Belin, 2003: 47-61.

«Notes, questions et discussions», Revue de Synthèse historique, I/1 (Paris, agosto 1900): 111-113. 
«Notes, questions et discussions», Revue de Synthèse historique, X-1/28 (Paris, agosto 1905): 369-372.

Oexle, Otto Gerhard (ed.), Krise des Historismus - Krise der Wirklichkeit, Wissenschaft, Kunst und Literatur 1880-1932, Göttingen, Vandenhoeck \& Ruprecht, 2007.

Pirenne, Henri, «Une polémique historique en Allemagne», Revue historique, 22éme année/LXIV (Paris, mayo-agosto 1896): 50-57.

«Préface», L’Année sociologique, I année (1896-1897), Paris, Félix Alcan, 1898: I-VII.

Prochasson, Christophe, «Histoire et Sociologie: Henri Berr et les durkheimiens (1900-1914)», Revue de Synthése, 117/1-2 (Paris, marzo 1996): 61-79.

Prost, Antoine, «Charles Seignobos revisité», Vingtième siècle, 43 (Paris, julioseptiembre 1994): 100-118.

Rachfahl, Felix, «Deutsche Geschichte vom wirtschaftliche Standpunk», Preußische Jahrbücher, 83 (Berlin, 1896): 48-96.

Revel, Jacques, «Le moment Berr», Revue de Synthése, 117/1-2 (Paris, marzo 1996): 157-172.

Revue de Synthèse historique, III-3/9 (Paris, diciembre 1901): 350-357.

Revue de Synthèse historique, IV-1/10 (Paris, febrero 1902): 107-109.

Rheinberger, Hans-Jörg, Toward a History of Epistemic Things. Synthesizing Proteins in the Test Tube, Stanford, Stanford University Press, 1997.

Rheinberger, Hans-Jörg, «Consistency from the Perspective of an Experimental Systems Approach to the Sciences and their Epistemic Objects», Manuscrito. Revista Internacional de Filosofía, 34/1 (São Paulo, enero-junio 2011): 307-321.

Rickert, Heinrich, Kulturwissenschaft und Naturwissenschatf. Ein Vortrag, Freiburg am Brisgau, Leipzig, Tübingen, J. C. B. Mohr, 1899 (trad. al español de la $4^{\mathrm{a}}$ y $5^{\mathrm{a}}$ edición alemana ampliada: Ciencia de la cultura y ciencia natural, Madrid, Calpe, 1922).

Rickert, Heinrich, «Les quatre modes de l' «Universel» en histoire», Revue de Synthèse historique, II-2/5 (Paris, abril 1901): 121-140.

Ringer, Fritz K., The Decline of the German Mandarins: the German Academic Community 1890-1933, Cambridge, Harvard University Press, 1969 (trad. al esp.: El ocaso de los mandarines alemanes. La comunidad académica alemana 18901933, Barcelona, Pomares-Corredor, 1995).

Schlanger, Judith, «Fondation, nouveauté, limites, mémoire», Communications, 54 (Paris, 1992): 291-292.

Schmoller, Gustav, «Zur Methodologie der Staats- und Sozialwissenschaften», Jahrbuch für Gesetzgebung, Verwaltung und Volkswittschaft im deutschen Reich, 7 (Leipzig, 1883): 239-258.

Schmoller, Gustav, Política social y economía política, 2 vols., Barcelona, Imp. Henrich y Comp ${ }^{\mathrm{a}}$ en C., 1905 (obra reed. en Granada, Comares, 2007).

Schorn-Schütte, Luise, Karl Lamprecht. Kulturgeschichtsschreibung zwischen Wissenschaft und Politik, Göttingen, Vandenhoeck \& Ruprecht, 1984.

Seignobos, Charles, «L'enseignament de l'histoire dans les universités allemandes», Revue internationale de l'enseignemente, I, Paris, 1881. 
Seignobos, Charles, La Méthode historique appliquée aux sciences sociales, Paris, Félix Alcan, 1901 (puede verse una reedición reciente electrónica, con prefacio de Antoine Prost, en Lyon, ENS Éditions, 2014 [http://books.openedition.org/enseditions/487]). Trad. al esp.: El método histórico aplicado a las ciencias sociales, Madrid, Daniel Jorro editor, 1923.

Seignobos, Charles y Bouglé, Célestine, «Rapports de la Sociologie avec l’Histoire», Revue international de Sociologie, XIIème année (Paris, 1904): 161-167.

Seignobos, Charles, «Les conditions pratiques de la recherche des causes dans le travail historique», Bulletin de la Société française de philosophie, VII (Paris, 1907): 261-299.

Seignobos, Charles, «L'inconnu et l'inconscient en histoire», Bulletin de la Société française de philosophie, VIII (Paris, 1908): 217-247.

Simiand, François, Revue de méthaphysique et morale, VI (Paris, 1898): 632-641.

Simiand, François, «Méthode historique et Science sociale. Étude critique d'après les ouvrages récents de M. Lacombe et de M. Seignobos», Revue de Synthèse historique, VI-1/16 (Paris, febrero 1903): 1-22

Simiand, François, «Méthode historique et Science sociale. Étude critique d'après les ouvrages récents de M. Lacombe et de M. Seignobos», Revue de Synthèse historique, VI-2/17 (Paris, abril 1903): 129-157.

Simiand, François, «Séance du 3 janvier 1903», Bulletin de la Société d'Histoire moderne, 2 (Paris, 1903a): 73-77.

Simiand, François, «Séance du 7 février 1903», Bulletin de la Société d'Histoire moderne, 2 (Paris, 1903b): 79.

Simiand, François, «La causalité en histoire», Bulletin de la Société française de philosophie, VI (Paris, 1906): 245-290.

Steiner, Philippe, L'école durkheimienne et l'économie, Gèneve, DROZ, 2005.

«Sur notre programme», Revue de Synthèse historique, I/1 (Paris, agosto 1900): 1-8.

Thomason, Burke C., Making Sense of Reification: Alfred Schutz and the Constructionist Theory, Londres, MacMillan, 1982.

Vogt, W. Paul, «Un durkheimien ambivalent: Célestin Bouglé, 1870-1940)», Revue française de sociologie, 20/1 (Paris, 1979): 123-139.

Recibido: 20/02/2017

Aprobado: 04/07/2017 